\title{
Vortices on the cylinder
}

\author{
Urs Frauenfelder*
}

November 10, 2018

\begin{abstract}
We apply the finite dimensional approximation techniques of Furuta, Kronheimer, and Manolescu to give a new proof of a result of Jaffe and Taubes.
\end{abstract}

\section{Contents}

\begin{tabular}{lll}
\hline & Introduction & 2
\end{tabular}

$\begin{array}{|ll|}2 \text { Proof of Theorem A } & 4\end{array}$

2.1 The gradient equation . . . . . . . . . . . . . . . . 4

2.2 Finite dimensional approximation $\ldots \ldots \ldots \ldots \ldots$

2.3 The maps of Jaffe and Taubes . . . . . . . . . . . . . . . . 12

3 Further directions 15

3.1 The svmplectic vortex equations and Givental's toric map spaces 15

3.2 Warped product metrics and Chern-Simons Vortices . . . . . . . 17

\begin{tabular}{|l|l|}
\hline A Morse functions with Lagrange multipliers & 18
\end{tabular}

*Supported by JSPS 


\section{Introduction}

The vortex equations are the absolute minima of the Yang-Mills-Higgs functional. For a unitary line bundle $L$ over a Riemann surface let $\mathfrak{A}$ be the space of unitary connections of $L$ and $\Omega(L)$ be the space of smooth sections of $L$ then the Yang-Mills-Higgs functional $Y M H: \mathfrak{A} \times \Omega(L) \rightarrow \mathbb{R}$ is defined as

$$
Y M H(A, v)=\int_{\Sigma}\left(\left|F_{A}\right|^{2}+\left|\nabla_{A} v\right|^{2}+\frac{1}{4}\left(1-|v|^{2}\right)^{2}\right) d v o l .
$$

Its absolute minima

$$
\begin{array}{r}
\bar{\partial}_{A} v=0 \\
* F_{A}=\frac{1}{2}\left(|v|^{2}-1\right)
\end{array}
$$

are the vortex equations. The Yang-Mills-Higgs functional is invariant under the action of the gauge group $\mathcal{G}=C^{\infty}\left(\Sigma, S^{1}\right)$ and hence so are the vortex equations.

For the case $\Sigma=\mathbb{C}$ the moduli spaces for the vortex equations were completely described by Jaffe and Taubes, see [17. For solutions $(A, v)$ of the vortex equations on $\mathbb{C}$ satisfying an appropriate decay condition at infinity, it turns out that the vortex number

$$
N=\frac{1}{2 \pi} \int_{\mathbb{C}} F_{A}
$$

is an integer. Jaffe and Taubes proved that the moduli space of vortices with vortex number $N$ modulo gauge is given by the $N$-fold symmetric product

$$
\{\text { (1) : vortex number }=N\} / \mathcal{G} \cong S^{N} \mathbb{C} \cong \mathbb{C}^{N} \text {. }
$$

The case for compact Riemann surfaces $\Sigma$ was studied by Bradlow and GarciaPrada, see [1, 10]. In the compact case the vortex number is

$$
N=\left\langle c_{1}(L),[\Sigma]\right\rangle
$$

and the moduli space was determined by Bradlow and Garcia-Prada

$$
\{\text { (11) : vortex number }=N\} / \mathcal{G} \cong\left\{\begin{array}{cl}
S^{N} \Sigma & N<\operatorname{vol}(\Sigma) / 4 \pi \\
\emptyset & N>\operatorname{vol}(\Sigma) / 4 \pi
\end{array}\right.
$$

If $N=\operatorname{vol}(\Sigma) / 4 \pi$ then solutions of (1) necessarily satisfy $v \equiv 0$. In this article we consider the case where $\Sigma=\mathcal{Z}$ is the cylinder. We prove

Theorem A The moduli space $N$-vortices on the cylinder modulo gauge is $S^{N} \mathcal{Z}$.

We do not claim originality for this theorem since the methods of Jaffe and Taubes for the complex plane could also be used to determine the vortices on the cylinder. However, we will present in this paper a new approach for proving existence of PDE's by using the finite dimensional approximation techniques of 
Furuta, Kronheimer, and Manolescu [9, 20, 23].

The idea of this new method is the following. Solutions of the vortex equations on the cylinder can be interpreted as flow lines of an action functional $\mathcal{A}$ defined on an infinite dimensional space $\mathscr{L}$. We consider a finite dimensional approximation $L \subset \mathscr{L}$ and homotop the flow lines of $\mathcal{A}$ to the flow lines of $\left.\mathcal{A}\right|_{L}$. Since $L$ is finite dimensional the flow lines of the restricted action functional are solutions of an ODE. This enables us to translate the question of existence of a PDE to the question of existence of an ODE.

However, to prove existence of finite energy Morse flow lines on a noncompact manifold is still a hard task. To do that we will take advantage of the fact that the restriction of our action functional to the finite dimensional approximation has the form of a Lagrange multiplier functional. It is well known from basic calculus that critical points of a function under a constraint can be found by considering the Lagrange multiplier functional. However, the Morse flow lines of the Lagrange multiplier functional and the Morse flow lines of the function restricted to the constraint are in general quite different. We will develop a theory which shows how they can be homotoped to each other. This theory allows us to translate the question of existence of Morse flow lines on a noncompact manifold to the question of existence of Morse flow lines on a compact manifold.

This paper is organized as follows. In Section 2 we prove Theorem A using finite dimensional approximation modulo the theory of Morse functions with Lagrange multipliers. In Section 3 we discuss further examples were our methods could be applied. In the Appendix we discuss the Theory of Morse functions with Lagrange multipliers.

Acknowledgements: I would like to thank Kaoru Ono for pointing out to me that the finite dimensional approximation techniques of Furuta, Kronheimer, and Manolescu can be applied to the vortex equations on the cylinder. The final part of this paper was written at FIM of ETH Zürich. I wish to thank FIM for its kind hospitality. 


\section{Proof of Theorem A}

\subsection{The gradient equation}

The standard circle action on $\mathbb{C}$ given by

$$
z \mapsto e^{i \theta} z, \quad e^{i \theta} \in S^{1}
$$

is Hamiltonian with respect to the standard symplectic structure $\omega=d x \wedge d y$ on $\mathbb{C}$. A moment map for the action is given by

$$
\mu(z)=-\frac{i}{2}|z|^{2}+\frac{i}{2} \in i \mathbb{R}=\operatorname{Lie}\left(S^{1}\right) .
$$

We consider the loop space

$$
\mathscr{L}=C^{\infty}\left(S^{1}, \mathbb{C} \times i \mathbb{R}\right)
$$

and define the action functional $\mathcal{A}: \mathscr{L} \rightarrow \mathbb{R}$ by

$$
\mathcal{A}(v, \eta)=\int_{0}^{1} \lambda(v)\left(\partial_{t} v\right)+\int_{0}^{1}\langle\mu(v), \eta\rangle d t
$$

where $\lambda=y d x$ is the Liouville one-form on $\mathbb{C}$ satisfying $d \lambda=-\omega$. The first term in (22) is just Floer's action functional on the loop space $C^{\infty}\left(S^{1}, \mathbb{C}\right)$,

$$
\mathcal{A}_{f l}(v)=\int_{0}^{1} \lambda(v)\left(\partial_{t} v\right)
$$

where the second term may be thought of as a Lagrange multiplier to the constraint $\mu^{-1}(0)$.

The gauge group

$$
\mathcal{H}=C^{\infty}\left(S^{1}, S^{1}\right)
$$

acts on $(v, \eta) \in \mathscr{L}$ by

$$
h_{*}(v, \eta)=\left(h v, \eta-h^{-1} \partial_{t} h\right), \quad h \in \mathcal{H} .
$$

The differential of the action functional $d \mathcal{A}$ and the $L^{2}$-metric $g_{L^{2}}$ on $\mathscr{L}$ are invariant under the gauge action and hence also the gradient flow lines of $\nabla_{g_{L^{2}}} \mathcal{A}$ which are solutions $(v, \eta) \in C^{\infty}\left(\mathbb{R} \times S^{1}, \mathbb{C} \times i \mathbb{R}\right)$ of the following PDE

$$
\begin{array}{r}
\partial_{s} v+i \partial_{t} v+i \eta v=0 \\
\partial_{s} \eta+\mu(v)=0 .
\end{array}
$$

Solutions of (3) are solutions of (11) in radial gauge. One has a natural bijection

$$
\{\text { (1) }\} / \mathcal{G} \cong\{\text { (3) }\} / \mathcal{H}
$$

by setting $A=\eta d t+\zeta d s$ and using a gauge transformation $g \in \mathcal{G}$ such that $g_{*} \zeta=0$. 
Remark 2.1 There is a straightforward generalization of the action functional (2) to general Hamiltonian group actions on symplectic manifolds. The gradient flow lines of these action functionals are the symplectic vortex equations [2, 3, 7] which reduce in the special case of a circle action on 1-dimensional complex space to the classical vortex equations.

Since the Marsden-Weinstein quotient $\mu^{-1}(0) / S^{1}$ is just a point, the critical manifold of $\mathcal{A}$ is homeomorphic to the gauge group $\mathcal{H}$. In particular,

$$
\pi_{0}(\operatorname{crit}(\mathcal{A})) \cong \pi_{0}(\mathcal{H}) \cong \pi_{1}\left(S^{1}\right) \cong \mathbb{Z}
$$

which enables us to recover the vortex number in this setting. The vortex number of a solution of (3) is proportional to the energy via

$$
N=\frac{1}{\pi} \lim _{s \rightarrow \infty}(\mathcal{A}((v, \eta)(s, \cdot))-\mathcal{A}((v, \eta)(-s, \cdot)))=\frac{1}{\pi} E(v, \eta)
$$

We denote by $\mathfrak{V}^{N}$ the moduli space of $N$-vortices on the cylinder, i.e. gradient flow lines of $\nabla_{g_{L_{2}}} \mathcal{A}$ modulo gauge which converge at the ends to connected components of $\operatorname{crit}(\mathcal{A})$ of difference $N \in \mathbb{Z}$. The following theorems follow from the results in [2].

Theorem 2.2 (Regularity) The moduli spaces $\mathfrak{V}^{N}$ are smooth manifolds of dimension $2 N$.

Theorem 2.3 (Compactness moduli breaking) Let $\left(v^{\nu}, \eta^{\nu}\right)$ be a sequence of $N$-vortices. Then there exists a subsequence $\nu_{j}$, a sequence of gauge transformations $h_{j} \in \mathcal{H}, N_{i}$-vortices $\left(v_{i}, \eta_{i}\right)$ for $1 \leq i \leq \ell$, and sequences of real numbers $S_{i}^{j}$ such that the timeshifted vortices converge uniformly in the $C_{\text {loc }}^{\infty}$-topology

$$
\left(h_{j}\right)_{*}\left(v^{\nu_{j}}, \eta^{\nu_{j}}\right)\left(\cdot, \cdot+S_{i}^{j}\right) \longrightarrow_{j \rightarrow \infty}\left(v_{i}, \eta_{i}\right)
$$

different timeshifts diverge

$$
\lim _{j \rightarrow \infty}\left|S_{i}^{j}-S_{i^{\prime}}^{j}\right|=\infty, \quad i \neq i^{\prime}
$$

and the total vortex number is preserved

$$
\sum_{i=1}^{\ell} N_{i}=N
$$

\subsection{Finite dimensional approximation}

The gauge group decomposes

$$
\mathcal{H}=\mathcal{H}_{0} \oplus S^{1} \oplus \mathbb{Z} .
$$

where the infinite dimensional contractible group $\mathcal{H}_{0}$ is given by

$$
\mathcal{H}_{0}=\left\{g=\exp (\xi) \in \mathcal{H}: \xi \in C^{\infty}\left(S^{1}, i \mathbb{R}\right), \int_{0}^{1} \xi d t=0\right\} .
$$


Following Manolescu [23] we get rid of $\mathcal{H}_{0}$ by projecting the gradient equations to the Coulomb section in $\mathscr{L}$. To see how this works, observe that $\mathcal{H}_{0}$ acts freely on $\mathscr{L}$ and since our gauge group is abelian we can put each $\eta \in C^{\infty}\left(S^{1}, i \mathbb{R}\right)$ into global Coulomb gauge on the circle, namely there exists a unique $h_{\eta} \in \mathcal{H}_{0}$ such that

$$
0=d^{*}\left(\left(h_{\eta}\right)_{*} \eta\right)=-\partial_{t}\left(\left(h_{\eta}\right)_{*} \eta\right) .
$$

Hence we may think of

$$
\mathscr{L}_{c}=C^{\infty}\left(S^{1}, \mathbb{C}\right) \times i \mathbb{R}
$$

as a section in the principal $\mathcal{H}_{0}$-bundle $\mathscr{L}$, or more precisely, we have a commutative diagram

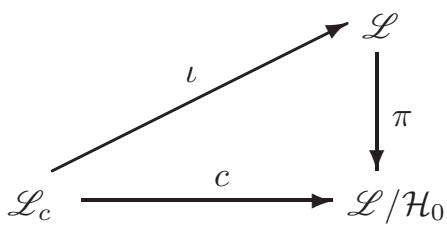

where $\iota$ denotes the canonical inclusion and $c$ denotes the bijection which is induced by Coulomb gauge.

The $L^{2}$-metric $g_{L^{2}}$ on $\mathscr{L}$ induces two natural metrics on $\mathscr{L}_{c}$

$$
g_{0}=\iota^{*} g_{L^{2}}, \quad g_{1}=c^{*}\left[g_{L^{2}}\right]
$$

where $\left[g_{L^{2}}\right]$ denotes the quotient metric of the $L^{2}$-metric on $\mathscr{L} / \mathcal{H}_{0}$.

Abbreviate

$$
\mathcal{A}_{c}=\left.\mathcal{A}\right|_{\mathscr{L}_{c}} .
$$

Then $\mathcal{H}_{0}$-gauge equivalence classes of flow lines of $\nabla_{g_{L^{2}}} \mathcal{A}$ are in natural oneto-one correspondence with flow lines of $\nabla_{g_{1}} \mathcal{A}_{c}$ by projection. The importance of $g_{0}$ lies in the fact that flow lines of $\nabla_{g_{0}} \mathcal{A}_{c}$ are contained in natural finite dimensional subspaces of the infinite dimensional space $\mathscr{L}_{c}$. For integers $\mu \leq \nu$ consider the Fourierapproximations

$$
L_{\mu}^{\nu}=\left\{z=\sum_{j=\mu}^{\nu} z_{j} e^{2 \pi i j}: z_{j} \in \mathbb{C}\right\}
$$

of the loop space $C^{\infty}\left(S^{1}, \mathbb{C}\right)$. The metric $g_{0}$ is just the product of the $L^{2}$-metric on $C^{\infty}\left(S^{1}, \mathbb{C}\right)$ and the metric induced from the inner product on $i \mathbb{R}$ and hence

$$
\nabla_{g_{0}} \mathcal{A}_{c}(z, \eta) \in L_{\mu}^{\nu} \times i \mathbb{R} \subset \mathscr{L}_{c}, \quad(z, \eta) \in L_{\mu}^{\nu} \times i \mathbb{R} .
$$

It follows that flow lines of $\left.\mathcal{A}_{c}\right|_{L_{\mu}^{\nu} \times i \mathbb{R}}$ are actually flow lines of $\mathcal{A}_{c}$. Moreover, critical points of $\mathcal{A}_{c}$ are tuples

$$
\left(v_{0} e^{2 \pi i m t}, 2 \pi m i\right), \quad\left|v_{0}\right|=1, m \in \mathbb{Z}
$$


and hence for every pair of critical points of $\mathcal{A}_{c}$ there exists a finite dimensional approximation as above which contains both of them.

The following proposition shows that for each finite energy flow line of $\mathcal{A}_{c}$ one can find a finite dimensional approximation such that the flow line is entirely contained in it. For a set of flow lines which converge at both ends to the same critical points there can be found a finite dimensional approximation which contains the whole set simultanuously. However note, that since there are infinitely many critical points of $\mathcal{A}_{c}$ there is no finite dimensional approximation in which all finite energy flow lines lie simultanuously.

Proposition 2.4 Assume that $(v, \eta) \in C^{\infty}\left(\mathbb{R} \times S^{1}, \mathbb{C}\right) \times C^{\infty}(\mathbb{R}, i \mathbb{R})$ is a gradient flow line of $\nabla_{g_{0}} \mathcal{A}_{c}$ such that

$$
\lim _{s \rightarrow \pm \infty}(v, \eta)(s, t)=\left(v_{ \pm} e^{2 \pi i m_{ \pm} t}, 2 \pi m_{ \pm} i\right), \quad\left|v_{ \pm}\right|=1, m_{ \pm} \in \mathbb{Z}
$$

where the limit is uniformly with respect to the $C^{\infty}$-topology. Then $(v, \eta)(s, \cdot)$ is contained in $L_{-m_{-}}^{-m_{+}} \times i \mathbb{R}$.

Proof: Abbreviate $\bar{\mu}(v) \in C^{\infty}(\mathbb{R}, i \mathbb{R})$ by

$$
\bar{\mu}(v)(s)=\int_{0}^{1} \mu(v(s, t)) d t, \quad s \in \mathbb{R} .
$$

A gradient flow line of $\nabla_{g_{0}} \mathcal{A}_{c}$ is a solution of the following PDE

$$
\begin{array}{r}
\partial_{s} v+i \partial_{t} v+i \eta v=0 \\
\partial_{s} \eta+\bar{\mu}(v)=0 .
\end{array}
$$

Plugging in the Fourierexpansion

$$
v(s, t)=\sum_{m=-\infty}^{\infty} v_{j}(s) e^{2 \pi i j t}
$$

into the first equation of (4) we obtain for each Fouriercoefficient the ODE

$$
\partial_{s} v_{m}(s)+(i \eta(s)-2 \pi m) v_{m}(s)=0 .
$$

Using (5), $\lim _{s \rightarrow \pm \infty} \partial_{s} v_{m}(s)=0$, and the asymptotic behaviour of $\eta(s)$, we conclude that $v_{m}$ vanishes identically unless $m$ is contained in $\left\{-m_{-}, \ldots,-m_{+}\right\}$. This proves the proposition.

In order to homotop the PDE (3) to an ODE it remains in view of the proposition above to find a homotopy between $g_{0}$ and $g_{1}$. This homotopy has to be compact, i.e. the moduli spaces of finite energy flow lines should be compact modulo breaking and modulo the remaining action of the noncompact group $\mathbb{Z}$. Moreover, we require the homotopy to be equivariant with respect to the following torus action. There is the circle action on the target manifold $\mathbb{C}$ and there 
is a further circle action on the domain $S^{1}$ given by rotating the circle. The two actions commute on $\mathscr{L}_{c}$ and lead to an action of the two torus $T^{2}=S^{1} \times S^{1}$ on $\mathscr{L}_{c}$. Note that the action functional $\mathcal{A}_{c}$, and the metrics $g_{0}$ and $g_{1}$ are $T^{2}$-invariant.

Theorem 2.5 There exists a continuous family of $T^{2}$-invariant metrics $g_{r}$ for $r \in[0,1]$ on $\mathscr{L}_{c}$ with the following property. Assume that for $\nu \in \mathbb{N}$ there exists a sequence of flow lines $\left(v^{\nu}, \eta^{\nu}\right)$ of $\nabla_{g_{r^{\nu}}} \mathcal{A}_{c}$ for $r^{\nu} \in[0,1]$ whose energy is uniformly bounded, i.e. there exists a constant $c>0$ such that for all $\nu$ it holds

$$
E\left(v^{\nu}, \eta^{\nu}\right) \leq c .
$$

Then there exists a subsequence $\nu_{j}$, a sequence of gauge transformations $h_{j} \in \mathbb{Z}$, flow lines $\left(v_{i}, \eta_{i}\right)$ for $1 \leq i \leq \ell$ of $\nabla_{g_{r_{\infty}}} \mathcal{A}_{c}$ for $r_{\infty} \in[0,1]$, and sequences of real numbers $S_{i}^{j}$ such that the timeshifted vortices converge uniformly in the $C_{\text {loc }}^{\infty}$ topology

$$
\left(h_{j}\right)_{*}\left(v^{\nu_{j}}, \eta^{\nu_{j}}\right)\left(\cdot, \cdot+S_{i}^{j}\right) \longrightarrow_{j \rightarrow \infty}\left(v_{i}, \eta_{i}\right)
$$

different timeshifts diverge

$$
\lim _{j \rightarrow \infty}\left|S_{i}^{j}-S_{i^{\prime}}^{j}\right|=\infty, \quad i \neq i^{\prime}
$$

and the total energy is preserved

$$
\lim _{j \rightarrow \infty} E\left(v^{\nu_{j}}, \eta^{\nu_{j}}\right)=\sum_{i=1}^{\ell} E\left(v_{i}, \eta_{i}\right)
$$

Proof: We first construct a $T^{2}$-invariant homotopy between $g_{0}$ and $g_{1}$. In order to do that, observe that the geometric reason that $g_{0}$ and $g_{1}$ are different lies in the fact that the infinitesimal gauge action of $\mathcal{H}_{0}$ is not orthogonal to the Coulomb section $\mathscr{L}_{c}$ with respect to the $L^{2}$-metric on $\mathscr{L}$. To construct the homotopy we consider a family of $\mathcal{H}_{0}$ actions on $\mathscr{L}$ such that $\mathscr{L}_{c}$ is a section for the whole family of actions but that for the final action the Coulomb section gets orthogonal to the infinitesimal gauge action.

Taking advantage of the contractibility of the gauge group $\mathcal{H}_{0}$ we define for $r \in[0,1]$ and $h=\exp (\xi) \in \mathcal{H}_{0}$ the $h_{*_{r}}$ action on $(v, \eta) \in \mathscr{L}$ by

$$
h_{*_{r}}(v, \eta)=\left(\exp (r \xi) v, \eta-h^{-1} \partial_{t} h\right) .
$$

The deformed actions of $\mathcal{H}_{0}$ are still free on $\mathscr{L}$ and $\mathscr{L}_{c}$ is a simultanuous section for the whole family of actions. For each $r \in[0,1]$ we have a commutative diagram

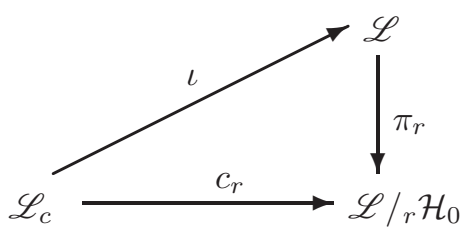


where $\mathscr{L} / r \mathcal{H}_{0}$ denotes the quotient of $\mathscr{L}$ under the $r$-action of $\mathcal{H}_{0}, \pi_{r}$ denotes the according canonical projection, and $c_{r}$ refers to the Coulomb gauge of the $r$-action. The $L^{2}$-metric on $\mathscr{L}$ is simultanuously $\mathcal{H}_{0}$ invariant for the whole family of actions and hence induces for every $r \in[0,1]$ a quotient metric $\left[g_{L^{2}}\right]_{r}$ on $\mathscr{L} / r \mathcal{H}_{0}$. We define

$$
g_{r}=c_{r}^{*}\left[g_{L^{2}}\right]_{r} .
$$

It is easy to check that $g_{r}$ are $T^{2}$-invariant for every $r \in[0,1]$. Moreover, for $r=0$ the Coulomb section is orthogonal to the 0 -action of $\mathcal{H}_{0}$ and hence $g_{0}$ defined in this way agrees with the previous definition of $g_{0}$.

The gradient flow lines of $\mathcal{A}_{c}$ with respect to the metric $g_{r}$ are solutions of the following problem

$$
\begin{aligned}
\partial_{s} v+\xi_{v} v+i \partial_{t} v+i \eta v & =0 \\
\partial_{s} \eta+\bar{\mu}(v) & =0
\end{aligned}
$$

where $\xi_{v} \in C^{\infty}\left(\mathbb{R} \times S^{1}, i \mathbb{R}\right)$ which is determined for every $s \in \mathbb{R}$ by the conditions

$$
\partial_{t} \xi_{v}(s, \cdot)=r^{2}(\mu(v(s, \cdot))-\bar{\mu}(v)(s)), \quad \int_{0}^{1} \xi_{v}(s, t) d t=0 .
$$

The main difficulty for proving the compactness statement in Theorem 2.5 is to obtain a uniform $L^{\infty}$-estimate independent of $r \in[0,1]$ for all finite energy solutions of (6). This provides the following lemma.

Lemma 2.6 Let $(v, \eta) \in C^{\infty}\left(S^{1} \times \mathbb{R}, \mathbb{C}\right) \times C^{\infty}(\mathbb{R}, i \mathbb{R})$ be a finite energy solution of (6) for $r \in[0,1]$. Then there exists a constant $c<\infty$ independent of $r$ such that $\|v\|_{\infty}<c$

Proof: Define $u(s)=\frac{1}{2} \int_{0}^{1}|v(s, t)|^{2} d t$ for $s \in \mathbb{R}$.

Step 1: $u(s) \leq 1 / 2$ for every $s \in \mathbb{R}$.

Using a computation similar to the one in the proof of [3, Proposition 3.5] we estimate

$$
\begin{aligned}
\partial_{s}^{2} u= & \int_{0}^{1}\left(\left|\partial_{s} v+\xi_{v} v\right|^{2}+\left|\partial_{t} v+\eta v\right|^{2}\right) d t+ \\
& 2 \int_{0}^{1}\left\langle\mu(v),\left(1-r^{2}\right) \bar{\mu}(v)+r^{2} \mu(v)-i / 2\right\rangle d t \\
\geq & 2\langle\bar{\mu}(v), \bar{\mu}(v)-i / 2\rangle \\
= & 2\langle u, u+1 / 2\rangle \\
\geq & 2 u(u-1 / 2) .
\end{aligned}
$$

Hence if $u\left(s_{0}\right)>1 / 2$ for $s_{0} \in \mathbb{R}$, then $u$ cannot have a local maximum at $s_{0}$. However the finite energy assumption implies that $\lim _{s \rightarrow \pm \infty} u(s)=1 / 2$ which 
proves Step 1.

Step 2: There exists a constant $c_{1}$ and a gauge transformation $h \in \mathbb{Z}$ such that $\left\|\partial_{t}\left(h_{*} v\right)\right\|_{\infty} \leq c_{1}\left\|h_{*} v\right\|_{\infty}^{2}=c_{1}\|v\|_{\infty}^{2}$.

Fix some integer $n>3$ and consider the finite cylinder $\mathcal{Z}_{n}=S^{1} \times[-n, n]$. It follows from Step 1 that

$$
\|v\|_{L^{2}\left(\mathcal{Z}_{n}\right)}=\mathcal{O}(1)
$$

After a gauge transformation we may assume without loss of generality that

$$
\|\eta(0)\|=\mathcal{O}(1) .
$$

Using the second equation in (6) and Step 1 we conclude that

$$
\|\eta\|_{L^{\infty}\left(\mathcal{Z}_{n}\right)}=\mathcal{O}(1) \text {. }
$$

The definition of $\xi_{v}$ together with Step 1 implies that

$$
\left\|\xi_{v}\right\|_{L^{\infty}\left(\mathcal{Z}_{n}\right)}=\mathcal{O}(1) .
$$

Combining (17), (8), and (9) and using the first equation in (6) we conclude that

$$
\|\bar{\partial} v\|_{L^{2}\left(\mathcal{Z}_{n}\right)}=\mathcal{O}(1)
$$

from which we deduce using (77) and elliptic regularity for the Cauchy-Riemann operator

$$
\|v\|_{W^{1,2}\left(\mathcal{Z}_{n-1}\right)}=\mathcal{O}(1) .
$$

It follows from Sobolev's embedding theorem that for every $p<\infty$ we have

$$
\|v\|_{L^{p}\left(\mathcal{Z}_{n-1}\right)}=\mathcal{O}_{p}(1)
$$

from which we deduce analogously as before

$$
\|v\|_{W^{1, p}\left(\mathcal{Z}_{n-2}\right)}=\mathcal{O}_{p}(1) .
$$

Using (12) and the definition of $\xi_{v}$ we conclude

$$
\left\|\xi_{v}\right\|_{W^{1, p}\left(\mathcal{Z}_{n-2}\right)}=\mathcal{O}_{p}\left(\|v\|_{\infty}\right) .
$$

The Laplacian of $v$ satisfies the equation

$$
\begin{aligned}
\Delta v= & i\left(\partial_{t} \xi_{v}\right) v+i \xi_{v}\left(\partial_{t} v\right)-\left(\partial_{s} \xi_{v}\right) v-\xi_{v}\left(\partial_{s} v\right) \\
& -i\left(\partial_{s} \eta\right) v-i \eta\left(\partial_{s} v\right)-\eta\left(\partial_{t} v\right) .
\end{aligned}
$$

Using (13) and (12) we conclude from (14) that

$$
\|\Delta v\|_{L^{p}\left(\mathcal{Z}_{n-2}\right)}=\mathcal{O}_{p}\left(\|v\|_{\infty}^{2}\right)
$$


from which we conclude by elliptic regularity for the Laplace operator and (11)

$$
\|v\|_{W^{2, p}\left(\mathcal{Z}_{n-3}\right)}=\mathcal{O}_{p}\left(\|v\|_{\infty}^{2}\right) .
$$

Step 2 follows now from (15) and the Sobolev embedding theorem.

Step 3: Proof of the lemma.

Abbreviate $v_{s}=v(s, \cdot)$ and let $\left\|v_{s}\right\|_{p}$ be the $L^{p}$-norm on the circle. It follows from Step 1 and Step 2 that there exist constants $c_{0}$ and $c_{1}$ such that

$$
\left\|v_{s}\right\|_{2} \leq c_{0}, \quad\left\|\partial_{t} v_{s}\right\|_{\infty} \leq c_{1}\left\|v_{s}\right\|_{\infty}^{2}
$$

We may assume without loss of generality that

$$
\left|v_{s}(0)\right|=\left\|v_{s}\right\|_{\infty}
$$

We then estimate for $t \in S^{1}=\mathbb{R} / \mathbb{Z}$ using the second inequality in (16)

$$
\left|v_{s}(t)\right| \geq\left\|v_{s}\right\|_{\infty}-c_{1}\left\|v_{s}\right\|_{\infty}^{2}|t| .
$$

Hence

$$
\begin{aligned}
\left\|v_{s}\right\|_{2} & \geq\left(2 \int_{0}^{1 /\left(c_{1}\left\|v_{s}\right\|_{\infty}\right)} c_{1}^{2}\left\|v_{s}\right\|_{\infty}^{4} t^{2} d t\right)^{1 / 2} \\
& =\sqrt{\frac{2}{3 c_{1}}}\left\|v_{s}\right\|_{\infty}^{1 / 2}
\end{aligned}
$$

from which we deduce using the second inequality in (16)

$$
\left\|v_{s}\right\|_{\infty} \leq \frac{3 c_{1}}{2} \cdot c_{0}^{2}
$$

This proves the lemma.

Proof of Theorem 2.5 continued: It follows from the previous lemma that for gradient flow lines of $\nabla_{g_{r}} \mathcal{A}_{c}$ the first factor $v$ remains in the compact 1-ball around 0 in the complex plane. Compactness modulo breaking can now be deduced from the results in 2]. However note, that their arguments simplify in our case. Since our gauge group is abelian we only need an easy version of Uhlenbeck's compactness theorem. Moreover, the bubbling analysis can be avoided for the standard symplectic structure on $\mathbb{C}$ by using the elliptic estimate

$$
\|v\|_{W^{1,2}\left([-N, N] \times S^{1}\right)} \leq c_{N}\left(\|\bar{\partial} v\|_{L^{2}\left([-N-1, N+1] \times S^{1}\right)}+\|v\|_{L^{2}\left([-N-1, N+1] \times S^{1}\right)}\right)
$$

for every $N \in \mathbb{N}$ and a constant $c_{N}>0$. 


\subsection{The maps of Jaffe and Taubes}

In [17] Jaffe and Taubes defined a map from the moduli space of $N$-vortices on the complex plane to the $N$-fold symmetric product of the complex plane and showed that it is bijective. In this subsection we define the analogon of their map for the gradient flow lines of $\nabla_{g_{r}} \mathcal{A}_{c}$ for all $r \in[0,1]$. We prove that for $r=1$ the map is bijective. This proves Theorem A in the introduction. As a biproduct we will obtain the proof of the compactness statement in Theorem 2.5.

Denote by $\mathfrak{V}_{r}^{N}$ the moduli space of $N$-vortices with respect to the metric $g_{r}$. Then $\mathfrak{V}_{1}^{N}=\mathfrak{V}^{N}$ the moduli space introduced before. It is useful to write the map of Jaffe and Taubes from $\mathfrak{V}_{r}^{N}$ to the $N$-fold symmetric product of the cylinder as the composite of two maps. Denote by $\mathfrak{W}_{r}^{N}$ the space of distributions $w$ on the cylinder $\mathcal{Z}$ for which there exists $N$ not necessarily distinct points $z_{j} \in \mathcal{Z}$ such that $w$ is smooth outside of $\bigcup_{j=1}^{N}\left\{z_{j}\right\}$ on $\mathcal{Z}$ and satisfies the following integro Kazdan-Warner type problem with singularities and prescribed asymptotic behaviour

$$
\begin{aligned}
-\Delta w+r^{2} e^{w}+\left(1-r^{2}\right) \int_{0}^{1} e^{w} d t-1 & =-4 \pi \sum_{j=1}^{N} \delta\left(z-z_{j}\right) \\
\lim _{s \rightarrow \pm \infty} w(s, t) & =0
\end{aligned}
$$

where the limit is uniform with respect to the $t$-variable. Define the map $\mathfrak{T}_{r}^{N}: \mathfrak{V}_{r}^{N} \rightarrow \mathfrak{W}_{r}^{N}$ by

$$
\mathfrak{T}_{r}^{N}(v, \eta)=\ln |v|^{2}
$$

and the map $\mathfrak{J}_{r}^{N}: \mathfrak{W}_{r}^{N} \rightarrow S^{N} \mathcal{Z}$ by

$$
\mathfrak{J}_{r}^{N}(w)=\left[z_{1}, \ldots, z_{N}\right]
$$

Note that the composition $\mathfrak{J}_{r}^{N} \circ \mathfrak{T}_{r}^{N}$ maps a pair $(v, \eta)$ to the zeros of $v$ counted with multiplicity. For simplicity of notation we will often drop the index 1, i.e. $\mathfrak{J}^{N}$ means $\mathfrak{J}_{1}^{N}$, etc. We prove the following two theorems.

Theorem 2.7 For every $r \in[0,1]$ and every $N \in \mathbb{N}$ the map $\mathfrak{T}_{r}^{N}$ is bijective.

Theorem 2.8 For every $N \in \mathbb{N}$ the map $\mathfrak{J}^{N}=\mathfrak{J}_{1}^{N}$ is bijective.

As an easy corollary of the above two theorems we get Theorem A from the introduction.

Proof of Theorem A: By Theorem 2.7 and Theorem 2.8 the map $\mathfrak{J}^{N} \circ \mathfrak{T}^{N}$ gives a bijection for every $N \in \mathbb{N}$ between the moduli space of $N$-vortices on the cylinder and the $N$-fold symmetric product of the cylinder.

Remark 2.9 For $r=1$ the problem (18) simplifies to the following KazdanWarner type problem with singularities (see [19])

$$
\begin{aligned}
-\Delta w+e^{w}-1 & =-4 \pi \sum_{j=1}^{N} \delta\left(z-z_{j}\right) \\
\lim _{s \rightarrow \pm \infty} w(s, t) & =0 .
\end{aligned}
$$


The bijectivity in Theorem 2.8 means that the above problem has a unique solution. The hard part is to prove existence of a solution. Our existence proof is based on finite dimensional approximation.

Proof of Theorem 2.7. Note that for $r \in[0,1]$ the action functional $\mathcal{A}^{r}: \mathscr{L} \rightarrow \mathbb{R}$ defined by

$$
\mathcal{A}^{r}(v, \eta)=\int_{0}^{1} \lambda(v) \partial_{t} v+\int_{0}^{1}\langle r \mu(v(t))+(1-r) \bar{\mu}(v), \eta(t)\rangle d t
$$

is invariant under the $r$-action of $\mathcal{H}_{0}$ on $\mathscr{L}$ and

$$
\left.\mathcal{A}^{r}\right|_{\mathscr{L}_{c}}=\mathcal{A}_{c}
$$

It follows that the gradient flow of $\mathcal{A}^{r}$ with respect to the $L^{2}$-metric on $\mathscr{L}$ are in natural one-to-one correspondence with gradient flow lines of $\mathcal{A}_{c}$ with respect to the $g_{r}$-metric on $\mathscr{L}_{c}$ by projection on the Coulomb section. Note that projection on the Coulomb section does not change the value of $\ln |v|^{2}$ and hence we are left with showing the equivalence of flow line of $\nabla_{g_{L^{2}}} \mathcal{A}^{r}$ and solutions of the problem (18). Using the notation $\bar{\eta}=\int_{0}^{1} \eta(t) d t$ for $\eta \in C^{\infty}\left(S^{1}, i \mathbb{R}\right)$ gradient flow lines $(v, \eta) \in C^{\infty}(\mathcal{Z}, \mathbb{C} \times i \mathbb{R})$ of $\mathcal{A}^{r}$ with respect to the $L^{2}$-metric solve

$$
\begin{gathered}
\partial_{s} v+i \partial_{t} v+i r \eta v+i(1-r) \bar{\eta} v=0 \\
\partial_{s} \eta+r \mu(v)+(1-r) \bar{\mu}(v)=0 .
\end{gathered}
$$

It is now an easy exercise to show that $\mathfrak{T}_{r}^{N}$ is well-defined, i.e. $\ln |v|^{2}$ of solutions of (20) are solutions of the integro type Kazdan-Warner type problem with singularities, and that for each solution of (18) there exists a unique gauge equivalence class of $N$-vortices satisfying (20), for details see [17.

We finally embark on the prove of Theorem 2.8 We first prove a lemma.

Lemma 2.10 For every $N \in \mathbb{N}$ the map $\mathfrak{J}^{N}$ is injective and its image is open and closed in $S^{N} \mathcal{Z}$.

Proof: Assume that $w$ and $w^{\prime}$ are two solution of the problem (19) for the same $N$-tuple of singularities $\left[z_{1}, \ldots, z_{N}\right]$. Then its difference $w-w^{\prime}$ is asymptotically zero and $\Delta\left(w-w^{\prime}\right) \geq e^{w^{\prime}}\left(w-w^{\prime}\right)$. Hence $w=w^{\prime}$.

The map $\mathfrak{J}^{N} \circ \mathfrak{T}^{N}$ is a continuous, one-to-one map between manifolds of the same dimension. Hence it is open by the Invariance of domain theorem, see for example [13, Corollary 18.9].

To show that the image of $\mathfrak{J}^{N}$ is closed, assume that $w_{\nu} \in \mathfrak{W}^{N}$ is a sequence such that $z_{\nu}=\mathfrak{J}^{N}\left(w_{\nu}\right)$ converges to $z \in S^{N} \mathcal{Z}$ as $\nu$ goes to infinity. For the sequence of flow lines $v_{\nu}=\left(\mathfrak{T}^{N}\right)^{-1}\left(w_{\nu}\right)$ there exists a subsequence $v_{\nu_{j}}$ which converges to a broken flow line. But since $z_{\nu}$ converges, the limit broken flow line is actually unbroken. Hence $v_{\nu_{j}}$ converges to $v \in \mathfrak{V}^{N}$ and

$$
z=\mathfrak{J}^{N}\left(\mathfrak{T}^{N}(v)\right)
$$


Hence the image of $\mathfrak{J}^{N}$ is closed.

The main work lies in the following existence statement for 1-vortices.

Theorem 2.11 $\mathfrak{V}_{r}^{1}$ is not empty for every $r \in[0,1]$.

Proof: Consider the following cylinder action on the gradient flow lines

$$
(v, \eta)(s, t) \mapsto(v, \eta)(s+\sigma, t+\tau), \quad(\sigma, \tau) \in \mathcal{Z} .
$$

1-vortices cannot break and hence $\mathfrak{V}_{r}^{1} / \mathcal{Z}$ is compact by Theorem 2.5 for every $r \in[0,1]$. By Proposition 2.4 we can identify the flow lines of $\nabla_{g_{0}} \mathcal{A}_{c}$ describing 1-vortices with Morse-flow lines on the finite dimensional approximation $L_{0}^{1}$. The finite dimensional approximation $L_{0}^{1}$ can be identified with $\mathbb{C}^{2}$ via its Fourierbasis and the $T^{2}$-action is given by

$$
\left(e^{i \theta_{1}}, e^{i \theta_{2}}\right)\left(z_{1}, z_{2}\right) \mapsto\left(e^{i \theta_{1}} z_{1}, e^{i\left(\theta_{1}+\theta_{2}\right)} z_{2}\right) .
$$

Denote by $\mu_{L_{0}^{1}}$ the moment map of the circle action of the first factor in $S^{1} \times S^{1}=$ $T^{2}$ given by

$$
\mu_{L_{0}^{1}}\left(z_{1}, z_{2}\right)=-\frac{i}{2}\left(\left|z_{1}\right|^{2}+\left|z_{2}\right|^{2}\right)+\frac{i}{2} .
$$

The restriction of the action functional

$$
A=\left.\mathcal{A}_{c}\right|_{L_{0}^{1} \times i \mathbb{R}}=\left.\mathcal{A}\right|_{L_{0}^{1} \times i \mathbb{R}} \in C^{\infty}\left(L_{0}^{1} \times i \mathbb{R}\right)
$$

is given by

$$
A(v, \eta)=\mathcal{A}_{f l}(v)+\left\langle\mu_{L_{0}^{1}}(v), \eta\right\rangle .
$$

The moduli space $\mathfrak{V}_{0}^{1} / \mathcal{Z}$ can now be identied by Proposition 2.4 with the space of finite energy Morse flow lines of $A$ modulo $T^{2} \times \mathbb{R}$ where the group $\mathbb{R}$ acts by reparametrisation of flow lines.

The space $L_{0}^{1} \times i \mathbb{R}$ is finite dimensional but still noncompact. Using the results of Appendix $\mathrm{A}$ we can homotop our Morse flow lines further to Morse flow lines on a compact manifold. In order to do that note that the function $A$ on $L_{0}^{1} \times i \mathbb{R}$ is the Lagrange multiplier functional of

$$
H=\left.\mathcal{A}_{f l}\right|_{\mu_{L_{0}^{1}}^{-1}(0)} \in C^{\infty}\left(\mu_{L_{0}^{1}}^{-1}(0)\right) .
$$

Hence finite energy Morse flow lines of $A$ can be homotoped inside a compact subset of $L_{0}^{1} \times i \mathbb{R}$ to Morse flow lines of $H$. The manifold $\mu_{L_{0}^{1}}^{-1}(0)$ is the three sphere $S^{3}$, the circle action of the first factor in $T^{2}=S^{1} \times S^{1}$ is the Hopf fibration $S^{3} \rightarrow S^{2}$ and the circle action of the second factor in $T^{2}$ acts by rotation on the two-sphere $S^{2}$. The function $H$ induces on $S^{2}$ the height function. In particular, the action of $T^{2} \times \mathbb{R}$ on the Morse flow lines of $H$ is free and the quotient consists of exactly one point.

The upshot of our construction is that we can homotop the moduli space $\mathfrak{V}_{r}^{1} / \mathcal{Z}$ 
by a compact homotopy to a point. During this homotopy the Fredholm index is unchanged by Proposition A.2 We are now in position to show that $\mathfrak{V}_{r}^{1}$ is nonempty for every $r \in[0,1]$. Assume the contrary. Then we apply the abstract perturbation theory of [8, 21, 22, 24, 25] to our compact homotopy. Actually, since we do not have to compactify our moduli spaces by broken flow lines containing bubble trees, the more elementary theory of [4] is already sufficient. What we obtain is a compact branched manifold containing just one boundary point of weight one. But such an object does not exist. Hence $\mathfrak{V}_{r}^{1}$ is nonempty for every $r \in[0,1]$.

Proof of Theorem [2.8: It follows from Floer's gluing construction and Theorem 2.11 that $\mathfrak{V}^{N}$ is not empty for every $N \in \mathbb{N}$. Hence $\mathfrak{J}^{N}\left(\mathfrak{W}^{N}\right)=$ $\mathfrak{J}^{N} \circ \mathfrak{T}^{N}\left(\mathfrak{V}^{N}\right)$ is not empty in $S^{N} \mathcal{Z}$. Since the image im $\mathfrak{J}^{N}$ is open and closed by Lemma 2.10 and $S^{N} \mathcal{Z}$ is connected it follows that $\mathfrak{J}^{N}$ is surjective. Since again by Lemma $2.10 \mathfrak{J}^{N}$ is injective the theorem follows.

\section{$3 \quad$ Further directions}

\subsection{The symplectic vortex equations and Givental's toric map spaces}

Instead of the circle action on $\mathbb{C}$ we can study more generally linear torus actions on a complex vector space. Assume that for $k \leq n$ the torus $T^{k}=\left\{e^{i v}: v \in \mathbb{R}^{k}\right\}$ acts on the complex vector space $\mathbb{C}^{n}$ via the action

$$
\rho\left(e^{i v}\right) z=e^{i A v} z, \quad z \in \mathbb{C}^{n}, v \in \mathbb{R}^{k}
$$

for some $(n \times k)$-matrix $A$ with integer entries. We endow the Lie algebra of the torus

$$
\operatorname{Lie}\left(T^{k}\right)=\mathfrak{t}^{k}=i \mathbb{R}^{k}
$$

with its standard inner product. The action of the torus on $\mathbb{C}^{n}$ is Hamiltonian with respect to the standard symplectic structure $\omega=\sum_{i=1}^{n} d x_{i} \wedge d y_{i}$. Denoting by $A^{T}$ the transposed matrix of $A$ a moment map $\mu: \mathbb{C}^{n} \rightarrow \mathfrak{t}^{k}$ is given by

$$
\mu(z)=-i A^{T} w, \quad w=\frac{1}{2}\left(\begin{array}{c}
\left|z_{1}\right|^{2} \\
\vdots \\
\left|z_{n}\right|^{2}
\end{array}\right),
$$

i.e.

$$
d\langle\mu, \xi\rangle=\iota_{X_{\xi}} \omega, \quad \xi \in \mathfrak{t}^{k}
$$

for the vector field $X_{\xi}$ on $\mathbb{C}^{n}$ given by the infinitesimal action

$$
X_{\xi}(z)=\dot{\rho}(\xi)(z), \quad z \in \mathbb{C}^{n} .
$$

We assume the following hypothesis, 
(H) The moment map $\mu$ is proper and $T^{k}$ acts freely on $\mu^{-1}(\tau)$.

It follows from $(\mathrm{H})$ that the Marsden-Weinstein quotient

$$
\mathbb{C}^{n} / / T^{k}=\mu^{-1}(\tau) / T^{k}
$$

is a compact symplectic manifold of dimension

$$
\operatorname{dim}\left(\mathbb{C}^{n} / / T^{k}\right)=2(n-k),
$$

where the symplectic structure is induced from the standard symplectic structure on $\mathbb{C}^{n}$.

Let $\mathscr{L}$ be the loop space

$$
\mathscr{L}:=C^{\infty}\left(S^{1}, \mathbb{C}^{n} \times \mathfrak{t}^{k}\right) .
$$

The gauge group

$$
\mathcal{H}=C^{\infty}\left(S^{1}, T^{k}\right)
$$

acts on $\mathscr{L}$ by

$$
h_{*}(v, \eta)=\left(\rho(h) v, \eta-h^{-1} \partial_{t} h\right), \quad h \in \mathcal{H},(v, \eta) \in \mathscr{L} .
$$

Recall Floer's action functional $\mathcal{A}_{f l}: C^{\infty}\left(S^{1}, \mathbb{C}^{n}\right) \rightarrow \mathbb{R}$ given by

$$
\mathcal{A}_{f l}(v)=\int_{0}^{1} \lambda(v)\left(\partial_{t} v\right)
$$

where $\lambda$ denotes the Liouville 1 -form

$$
\lambda=\sum_{i=1}^{n} y_{i} d x_{i}, \quad d \lambda=-\omega .
$$

The Moment action functional $\mathcal{A}: \mathscr{L} \rightarrow \mathbb{R}$ is defined by

$$
\mathcal{A}(v, \eta):=\mathcal{A}_{f l}(v)+\int_{0}^{1}\langle\mu(v(t))-\tau, \eta(t)\rangle d t .
$$

Again one may think of $\eta$ in the second integral as a Lagrange multiplier. In particular, the critical points of $\mathcal{A}$ are the critical points of Floer's action on the constraint $\mu^{-1}(\tau)$.

The gradient flow lines of $\mathcal{A}$ with respect to the $L^{2}$-metric $g_{L^{2}}$ on $\mathscr{L}$ are solutions $(v, \eta) \in C^{\infty}\left(\mathbb{R} \times S^{1}, \mathbb{C}^{n} \times \mathfrak{t}^{k}\right)$ of

$$
\begin{array}{r}
\partial_{s} v+i \partial_{t} v+i \dot{\rho}(\eta) v=0 \\
\partial_{s} \eta+\mu(v)=\tau .
\end{array}
$$

These are examples of the symplectic vortex equations on the cylinder in temporal gauge. 
In this setting the symplectic vortex equations can be homotoped again via a Floer homotopy compact up to breaking of flow lines to Morse problems on finite dimensional compact manifolds. If one considers a finite dimensional Fourierapproximation $L$ of the loop space $C^{\infty}\left(S^{1}, \mathbb{C}^{n}\right)$ then the $T^{k}$-action on $\mathbb{C}^{n}$ induces a $T^{k}$-action on $L$ by coefficientwise multiplication. This action is again Hamiltonian with moment map $\mu_{L}$ normalized such that $\mu_{L}(0)=0$. The finite dimensional compact manifolds we end up with are

$$
G_{L}=\mu_{L}^{-1}(\tau) / T^{k}
$$

and the Morse function is again Floer's action functional restricted to $G_{L}$. The spaces $G_{L}$ are known as Givental's toric map spaces. They were introduced by Givental in [12] and studied by different authors in [11, 15, 28].

\subsection{Warped product metrics and Chern-Simons Vortices}

If we consider the same action functional $\mathcal{A}: \mathscr{L} \rightarrow \mathbb{R}$ as for the vortex equations but instead of the standard flat $L^{2}$-metric a warped product metric $g_{\gamma}$ for a smooth function $\gamma:[0, \infty) \rightarrow(0, \infty)$ given by

$$
g_{\gamma}(v, \eta)\left(\left(\hat{v}_{1}, \hat{\eta}_{1}\right),\left(\hat{v}_{2}, \hat{\eta}_{2}\right)\right)=\int_{0}^{1}\left\langle\hat{v}_{1}, \hat{v}_{2}\right\rangle d t+\int_{0}^{1} \gamma(|v|)^{2}\left\langle\hat{\eta}_{1}, \hat{\eta}_{2}\right\rangle d t
$$

for $(v, \eta) \in \mathscr{L}$ and $\left(\hat{v}_{1}, \hat{\eta}_{1}\right),\left(\hat{v}_{2}, \hat{\eta}_{2}\right) \in T_{(v, \eta)} \mathscr{L}$ we obtain the following gradient equations for $(v, \eta) \in C^{\infty}\left(\mathbb{R} \times S^{1}, \mathbb{C} \times i \mathbb{R}\right)$

$$
\begin{array}{r}
\partial_{s} v+i \partial_{t} v+i \eta v=0 \\
\partial_{s} \eta+\frac{1}{\gamma(|v|)^{2}} \mu(v)=0 .
\end{array}
$$

In particular, if we choose

$$
\gamma(r)=\frac{1}{r}
$$

we obtain

$$
\begin{gathered}
\partial_{s} v+i \partial_{t} v+i \eta v=0 \\
\partial_{s} \eta+|v|^{2} \mu(v)=0 .
\end{gathered}
$$

These are the selfduality equations for the Chern-Simons vortices on the cylinder discovered by Hong-Kim-Pac and Jackiw-Weinberg, see 14] 16]. We refer the reader to the excellent textbook of Y. Yang [29] for a detailed treatment of this equation. This textbook may also serve as a guide to the corresponding literature.

Note that for this choice of $\gamma$ the metric $\gamma_{v}$ becomes singular if $v$ goes to zero. One may think of this as a continuum of "critical points at infinity" for each $(0, \eta)$ where $\eta$ is a smooth loop in the Lie algebra $i \mathbb{R}$. In particular, the action functional $\mathcal{A}$ takes on the set of "critical points at infinity" every 
value in $\mathbb{R}$. The energy of flow lines which converge at one end to a "critical point at infinity" can therefore be any value in $\mathbb{R}$, in contrast to the classical vortex equations where the energy of finite energy flow lines was quantized. Such solutions are called in the physics literature "nontopological solutions". We again refer to the textbook of Y. Yang [29] and the literature cited therein for a detailed treatment of nontopological solutions. For compact Riemann surfaces existence of "nontopological solutions" was proved by Tarantello and Ding-Jost-Li-Peng-Wang [5] 27]. In this case the "nontopological solutions" are characterized by the property that $v$ converges to 0 under the adiabatic limit obtained by letting the Chern-Simons coupling parameter tend to zero.

On the finite dimensional approximations the Chern-Simons vortices are flow lines of a Lagrange multiplier functional with respect to a warped product metric. "Critical points at infinity" are responsible for the failure of the Palais-Smale condition discussed in the appendix. So the study of flow lines of Lagrange multiplier functionals with respect to a warped product metric is a finite dimensional analogon of the Abelian Chern-Simons-Higgs theory and should lead to a deeper understanding of the phenomenons occuring in this theory.

\section{A Morse functions with Lagrange multipliers}

Assume that $M$ is a finite dimensional manifold and $V$ is a finite dimensional real vector space. It is well known from basic calculus that critical points of a smooth function $f \in C^{\infty}(M)$ satisfying a constraint given by the zero set of a smooth function $h \in C^{\infty}(M, V)$ can be found by considering the Lagrange multiplier functional $F \in C^{\infty}\left(M \times V^{*}\right)$, where $V^{*}$ is the dual vector space of $V$, given by

$$
F\left(x, v^{*}\right)=f(x)+v^{*}(h(x)) .
$$

If 0 is a regular value of $h$ then there is a natural one-to-one correspondence between critical points of $F$ and critical points of $\left.f\right|_{h^{-1}(0)}$. However, Morse flow lines of $F$ and Morse flow lines of $\left.f\right|_{h^{-1}(0)}$ may be quite different. Even if $h^{-1}(0)$ is compact it is not a priori clear that the moduli spaces of flow lines of $F$ are compact modulo breaking since $F$ is neither bounded from above nor below. However, we will show that if $h$ is locally proper around 0 , then $F$ satisfies the Palais-Smale condition from which we can deduce that flow lines of $F$ remain in a compact subset of the noncompact manifold $M \times V^{*}$.

A first possibility to homotop Morse flow lines of $F$ to Morse flow lines of $\left.f\right|_{h^{-1}(0)}$ would be the adiabatic limit method. For a fixed Riemannian metric $g_{M}$ on $M$ and a fixed Riemannian metric $g_{V^{*}}$ on $V^{*}$, induced from a Euclidean scalar product on $V$ we consider the family of metrics $g_{\epsilon}$ on $M \times V^{*}$ for $\epsilon \in(0,1]$

$$
g_{\epsilon}=g_{M} \oplus \epsilon^{2} g_{V^{*}} .
$$

If $\epsilon$ goes to zero, then the gradient flow lines of $F$ with respect to the metric $g_{\epsilon}$ converge to gradient flow lines of $\left.f\right|_{h^{-1}(0)}$ with respect to the metric $\left.g_{M}\right|_{h^{-1}(0)}$. 
If a generalized implicit function theorem as in [6] can be established at $\epsilon=0$, then this would lead to a homotopy compact modulo breaking between the two moduli spaces of gradient flow lines.

In this section we will pursue another approach. We will consider a homotopy of $f$ and the Riemannian metric $g_{M}$ such that $\left.f\right|_{h^{-1}(0)}$ is unchanged during the homotopy but the normal derivatives of $\nabla_{g_{M}} f$ at $h^{-1}(0)$ are homotoped to zero. Since $\left.f\right|_{h^{-1}(0)}$ is fixed the critical points of the Lagrange multiplier functional can be canonically identified with the set of critical points of $\left.f\right|_{h^{-1}(0)}$ during the whole homotopy. If the normal derivatives of $\nabla_{g_{M}} f$ at $h^{-1}(0)$ vanish then the moduli space of Morse flow lines of $\left.f\right|_{h^{-1}(0)}$ is canonically contained in the moduli space of flow lines of $F$. We will prove that for special choices of $f$ and $g_{M}$ there are no other flow lines of $F$. The main idea is to choose $g_{M}$ in such a way that a tubular neighbourhood of $h^{-1}(0)$ in $M$ becomes very huge and then prove that finite energy flow lines have to remain in this tubular neighbourhood.

It is natural to formulate our main theorem in the language of Morse-Bott functions. In order to fix notation we recall briefly its definition. A function $F$ on a finite dimensional manifold $M$ is called Morse-Bott if the critical set is a submanifold of $M$ and for each $x \in \operatorname{crit}(F)$ we have

$$
T_{x} \operatorname{crit}(F)=\operatorname{ker} H_{F}(x)
$$

where $H_{F}(x)$ is the Hessian of $F$ at $x$. It is well known that the Morse-Bott condition implies that flow lines which remain in a compact set of $M$ converge at both ends exponentially fast to critical points of $F$. For a Riemannian metric $g$ on $M$ we denote by $\mathcal{M}(F, g)$ the moduli space of finite energy flow lines of $\nabla_{g} F$.

The main theorem of this section can now be statet in the following way.

Theorem A.1 Let $M$ be a finite dimensional manifold, $\Gamma$ be a Lie group acting on $M$, and let $(V,\langle\rangle$,$) be a finite dimensional Euclidean vector space. Assume$ that $g_{M}$ is a $\Gamma$-invariant, geodesically complete Riemannian metric on $M, h \in$ $C^{\infty}(M, V)$ and $f \in C^{\infty}(M)$ are $\Gamma$-invariant functions satisfying the following conditions.

- 0 is a regular value of $h$,

- $h$ is locally proper around 0 , i.e. there exists an open neighbourhood $V_{0}$ of 0 in $V$ such that $h^{-1}\left(\operatorname{cl}\left(V_{0}\right)\right)$ is compact,

- the restriction of $f$ to the compact manifold $h^{-1}(0)$ is Morse-Bott.

Denote by $V^{*}$ the dual vector space of $V$ and let $\Gamma$ act on $M \times V^{*}$ by $\gamma\left(x, v^{*}\right)=$ $\left(\gamma x, v^{*}\right)$ for $\gamma \in \Gamma$ and $\left(x, v^{*}\right) \in M \times V^{*}$. Then there exists a smooth family of $\Gamma$-invariant Morse-Bott functions $F_{r} \in C^{\infty}\left(M \times V^{*}\right)$ for $r \in[0,1]$ and a smooth family of $\Gamma$-invariant Riemannian metrics $g_{r}$ on $M \times V^{*}$ satisfying

$$
F_{0}\left(x, v^{*}\right)=f(x)+v^{*}(h(x)), \quad g_{0}=g_{M} \oplus g_{V^{*}}
$$


where $g_{V *}$ is the metric on $V^{*}$ induced from the scalar product $\langle$,$\rangle on V$, such that the following conditions are satisfied.

(i) The inclusion $\iota: h^{-1}(0) \rightarrow M \times V^{*}, x \mapsto(x, 0)$ induces a bijection

$$
\iota_{*}: \mathcal{M}\left(\left.f\right|_{h^{-1}(0)},\left.g_{M}\right|_{h^{-1}(0)}\right) \rightarrow \mathcal{M}\left(F_{1}, g_{1}\right)
$$

defined by

$$
\iota_{*} y(s)=\iota(y(s)), \quad y \in \mathcal{M}\left(\left.f\right|_{h^{-1}(0)},\left.g_{M}\right|_{h^{-1}(0)}\right), s \in \mathbb{R} .
$$

(ii) For $r \in[0,1]$ there exists a smooth family of diffeomorphism $\phi_{r}:$ crit $F_{0} \rightarrow$ crit $F_{r} \subset M \times V^{*}$.

(iii) There exists a compact set $K \subset M \times V^{*}$ such that

$$
\left\{y(\sigma): y \in \bigcup_{r \in[0,1]} \mathcal{M}\left(F_{r}, g_{r}\right), \sigma \in \mathbb{R}\right\} \subset K .
$$

Proof: We prove the theorem in seven steps.

Step 1 (Neighbourhood of the constraint): There exists an open neighbourhood $V_{1}$ of 0 in $V$, a $\Gamma$-invariant open neighbourhood $U$ of $h^{-1}(0)$ in $M$, and $a$ $\Gamma$-equivariant diffeomorphism

$$
\phi: h^{-1}(0) \times V_{1} \rightarrow U
$$

where $\Gamma$ acts on $h^{-1}(0) \times V_{1}$ by $\gamma(x, v)=(\gamma x, v)$ for $\gamma \in \Gamma$ and $(x, v) \in h^{-1}(0) \times$ $V_{1}$ such that

$$
h(\phi(x, v))=v, \quad(x, v) \in h^{-1}(0) \times V_{1} .
$$

Since 0 is a regular value of $h$ there exists an open neighbourhood $U_{0}$ of $h^{-1}(0)$ such that $d h(y)$ is surjectiv for every $y \in U_{0}$. For $v \in V$ define the vector field $\xi_{v}$ on $U_{0}$ by the conditions

$$
d h(y) \xi_{v}(y)=v, \quad \xi_{v}(y) \in \operatorname{ker} d h(y)^{\perp}, \quad y \in U_{0}
$$

where $\operatorname{ker} d h(y)^{\perp}$ denotes the orthogonal complement of the kernel of $d h(y)$ with respect to the metric $g_{M}$. Since $h^{-1}(0)$ is compact there exists an open neighbourhood $V_{1}$ of 0 in $V$ such that for each $v \in V_{1}$ and for each $x \in h^{-1}(0)$ there exists a unique solution $y_{x, v} \in C^{\infty}([0,1], U)$ of the problem

$$
y_{x, v}(0)=x, \quad \partial_{t} y_{x, v}(t)=\xi_{v}\left(y_{x, v}(t)\right), \quad t \in[0,1],
$$

and the map

$$
\phi(x, v)=y_{x, v}(1), \quad(x, v) \in h^{-1}(0) \times V_{1}
$$


is a diffeomorphism. Set $U=\phi\left(h^{-1}(0) \times V_{1}\right)$. Since $h$ and $g_{M}$ are $\Gamma$-invariant it follows that $\gamma\left(y_{x, v}\right)=y_{\gamma x, v}$ for $\gamma \in \Gamma$ and $(x, v) \in h^{-1}(0) \times V_{1}$. Hence $U$ is $\Gamma$ invariant and $\phi: h^{-1}(0) \times V_{1} \rightarrow U$ is a $\Gamma$-equivariant diffeomorphism. Moreover, we compute

$$
\begin{aligned}
h(\phi(x, v)) & =h\left(y_{x, v}(1)\right) \\
& =h\left(y_{x, v}(0)\right)+\int_{0}^{1} \frac{d}{d t} h\left(y_{x, v}(t)\right) d t \\
& =h(x)+\int_{0}^{1} d h\left(y_{x, v}(t)\right) \partial_{t} y_{x, v}(t) d t \\
& =\int_{0}^{1} d h\left(y_{x, v}(t)\right) \xi_{v}\left(y_{x, v}(t)\right) d t \\
& =\int_{0}^{1} v d t \\
& =v .
\end{aligned}
$$

This proves (22) and hence Step 1.

Step 2: We construct the homotopies.

In this step we construct a $\Gamma$-invariant function $f_{1} \in C^{\infty}(M)$ and a $\Gamma$-invariant metric $g_{M, 1}$ on $M$. We then set for $r \in[0,1]$

$$
f_{r}=(1-r) f+r f_{1}, \quad g_{M, r}=(1-r) g_{M}+r g_{M, 1}
$$

and define the homotopy of functions $F_{r} \in C^{\infty}\left(M \times V^{*}\right)$ by

$$
F_{r}\left(x, v^{*}\right)=f_{r}(x)+v^{*}(h(x)), \quad\left(x, v^{*}\right) \in M \times V^{*}
$$

and the homotopy of metrics $g_{r}$ on $M \times V^{*}$ by

$$
g_{r}=g_{M, r} \oplus g_{V^{*}} .
$$

Choose a small number $\delta>0$ such that the open $\delta$-ball $B_{\delta}=\{v \in V$ : $\|v\|<\delta\}$ is contained in the neighbourhood $V_{1}$ of 0 in $V$ constructed in Step 1. Moreover, since $h$ is locally proper at 0 , we may assume that

$$
h(x)>\delta \Rightarrow x \notin \phi\left(h^{-1}(0) \times B_{\delta}\right) .
$$

Choose further a cutoff function $\hat{\beta} \in C^{\infty}([0, \delta),[0,1])$ such that $\left.\hat{\beta}\right|_{[0, \delta / 2]}=1$ and $\left.\hat{\beta}\right|_{[3 \delta / 4, \delta)}=0$. Denote by $\pi_{1}: h^{-1}(0) \times B_{\delta} \rightarrow h^{-1}(0)$ and by $\pi_{2}: h^{-1}(0) \times B_{\delta} \rightarrow$ $B_{\delta}$ the projection to the first, respectively the second, factor. We will use the following $\Gamma$-invariant cutoff function on $M$ given by

$$
\beta(x)=\left\{\begin{array}{cc}
\beta\left(\left|\pi_{2}\left(\phi^{-1}(x)\right)\right|\right) & x \in \phi\left(h^{-1}(0) \times B_{\delta}\right) \\
0 & x \notin \phi\left(h^{-1}(0) \times B_{\delta}\right) .
\end{array}\right.
$$


Define the function $f_{1} \in C^{\infty}(M)$ by

$$
f_{1}(x)=\left\{\begin{array}{cc}
\beta(x) f\left(\pi_{1}\left(\phi^{-1}(x)\right)\right)+(1-\beta(x)) f(x) & x \in \phi\left(h^{-1}(0) \times B_{\delta}\right) \\
f(x) & x \notin \phi\left(h^{-1}(0) \times B_{\delta}\right) .
\end{array}\right.
$$

Set

$$
C:=\max _{x \in h^{-1}(0)}\{f(x)\}-\min _{x \in h^{-1}(0)}\{f(x)\}
$$

and choose a constant

$$
\kappa>\frac{16 C}{\delta^{2}} .
$$

Let $g_{\kappa}$ be the product metric on $h^{-1}(0) \times B_{\delta}$

$$
g_{\kappa}=\left.g_{M}\right|_{h^{-1}(0)} \oplus \kappa^{2} g_{B_{\delta}}
$$

where $g_{B_{\delta}}$ is the standard euclidean metric on the ball $B_{\delta} \subset V$. We are now able to define the metric $g_{M, 1}$ on $M$ by the formula

$$
g_{M, 1}(x)=\left\{\begin{array}{cc}
\beta(x)\left(\phi_{*} g_{\kappa}\right)(x)+(1-\beta(x)) g_{M}(x) & x \in \phi\left(h^{-1}(0) \times B_{\delta}\right) \\
g_{M}(x) & x \notin \phi\left(h^{-1}(0) \times B_{\delta}\right) .
\end{array}\right.
$$

Step 3: The trace of each finite energy flow line $y \in C^{\infty}\left(\mathbb{R}, M \times V^{*}\right)$ of $\nabla_{g_{1}} F_{1}$ is contained in $\phi\left(h^{-1}(0) \times B_{\delta / 2}\right) \times V^{*}$.

First note that if $x \in M \backslash \phi\left(h^{-1}(0) \times B_{\delta / 4}\right)$ and $v^{*} \in V^{*}$ then it follows from (22) and (23) that

$$
\left\|\nabla_{g_{1}} F_{1}\left(x, v^{*}\right)\right\| \geq\|h(x)\| \geq \frac{\delta}{4} .
$$

Observe further that the energy of a finite energy flow line is bounded from above by the constant $C$ introduced in (24), i.e.

$$
\int_{-\infty}^{\infty}\left\|\nabla_{g_{1}} F_{1}(y(s))\right\|_{g_{1}}^{2} d s \leq C .
$$

Now assume by contradiction that there exists $\sigma \in \mathbb{R}$ such that

$$
y(\sigma) \notin \phi\left(h^{-1}(0) \times B_{\delta / 2}\right) \times V^{*} .
$$

Denote by $\tau(\sigma)>\sigma$ the real number

$$
\tau(\sigma):=\min \left\{s \in \mathbb{R}: y(s) \notin \phi\left(h^{-1}(0) \times B_{\delta / 4}\right) \times V^{*}\right\} .
$$

Note that $\tau(\sigma)$ is finite, since the energy of the flow line $y$ is assumed to be finite and the critical points of $F_{1}$ lie in $h^{-1}(0) \times V^{*}$. Denoting by $\operatorname{dist}_{g_{1}}(\cdot, \cdot)$ 
the distance with respect to the metric $g_{1}$ we estimate using (25), (26), and (27)

$$
\begin{aligned}
C & <\frac{\delta^{2} \kappa}{16} \\
& \leq \frac{\delta}{4} \cdot \operatorname{dist}_{g_{1}}(y(\sigma), y(\tau(\sigma))) \\
& \leq \frac{\delta}{4} \int_{\sigma}^{\tau(\sigma)}\left\|\partial_{s} y(s)\right\|_{g_{1}} d s \\
& =\frac{\delta}{4} \int_{\sigma}^{\tau(\sigma)}\left\|\nabla_{g_{1}} F_{1}(y(s))\right\| d s \\
& \leq \int_{\sigma}^{\tau(\sigma)}\left\|\nabla_{g_{1}} F_{1}(y(s))\right\|^{2} d s \\
& \leq \int_{-\infty}^{\infty}\left\|\nabla_{g_{1}} F_{1}(y(s))\right\|^{2} d s \\
& \leq C .
\end{aligned}
$$

This contradiction shows that (28) cannot hold which proves Step 3.

Step 4: The trace of each finite energy flow line $y=\left(x, v^{*}\right) \in C^{\infty}\left(\mathbb{R}, M \times V^{*}\right)$ of $\nabla_{g_{1}} F_{1}$ is contained in $h^{-1}(0) \times\{0\}$.

It follows from Step 3 that $x$ is contained in the image of $\phi$. Denoting

$$
(q, w)=\phi^{-1}(x) \in C^{\infty}\left(\mathbb{R}, h^{-1}(0) \times B_{\delta}\right)
$$

we observe that the triple $\left(q, w, v^{*}\right)$ is a flow line of the function

$$
F\left(q, w, v^{*}\right)=f(q)+v^{*}(w)
$$

with respect to the metric

$$
\left.g_{M}\right|_{h^{-1}(0)} \oplus \kappa^{2} g_{B_{\delta}} \oplus g_{V^{*}} .
$$

Denote by $\Lambda: V^{*} \rightarrow V$ the isomorphism induced from the euclidean scalar product on $V$. Flow lines of $F$ are solutions of the following ODE

$$
\begin{aligned}
\partial_{s} q+\nabla_{\left.g_{M}\right|_{h^{-1}(0)}} f(q) & =0 \\
\partial_{s} w+\frac{1}{\kappa} \Lambda v^{*} & =0 \\
\partial_{s} v^{*}+\Lambda^{-1} w & =0 .
\end{aligned}
$$

It follows from the two last equations in (29) that there exist $w_{0}, w_{1} \in V$ such that

$$
w(s)=w_{0} \exp \left(\frac{s}{\sqrt{\kappa}}\right)+w_{1} \exp \left(-\frac{s}{\sqrt{\kappa}}\right)
$$


Since the energy of the flow line $y$ is assumed to be finite it follows that $w_{0}=$ $w_{1}=0$ and hence

$$
w(s)=0, \quad v^{*}(s)=0, \quad s \in \mathbb{R} .
$$

This proves Step 4 .

Step 5 (Uniform Palais-Smale condition): There exists a geodesically complete Riemannian metric $g_{P S}$ on $M \times V^{*}$, a compact set $K_{0} \subset M \times V^{*}$ and a constant $\epsilon>0$ such that for $y \in\left(M \times V^{*}\right) \backslash K_{0}$ and $r \in[0,1]$

$$
\nabla_{g r} F_{r}(y) \neq 0, \quad\left\|\nabla_{g_{r}} F_{r}(y)\right\|_{g_{r}}^{2} \geq \epsilon\left\|\nabla_{g_{r}} F_{r}(y)\right\|_{g_{P S}}
$$

where \|\|$_{g}$ denotes the norm induced from the metric $g$.

We choose $g_{P S}=g_{M} \oplus g_{V^{*}}=g_{0}$. Then $g_{P S}$ is geodesically complete by assumption. For $x \in M$ and $r \in[0,1]$ we denote by $d h(x)^{*_{r}}: V^{*} \rightarrow T_{x} M$ the adjoint of $d h(x)$ with respect to the inner products $g_{M, r}(x)$ on $T_{x} M$ and $\langle$, on $V^{*}$. With respect to the natural splitting $T_{\left(x, v^{*}\right)}(M \times V *) \cong T_{x} M \times V^{*}$ for $\left(x, v^{*}\right) \in M \times V^{*}$ the gradient of $F_{r}$ reads

$$
\begin{aligned}
\nabla_{g_{r}} F_{r}\left(x, v^{*}\right) & =\left(\begin{array}{c}
\nabla_{g_{M, r}} f_{r}(x)+\nabla_{g_{M, r}}\left(v^{*} \circ h\right)(x) \\
h(x)
\end{array}\right) \\
& =\left(\begin{array}{c}
\nabla_{g_{M, r}} f_{r}(x)+d h^{* r}(x) v^{*} \\
h(x)
\end{array}\right) .
\end{aligned}
$$

Since 0 is a regular value of $h$ and $h^{-1}\left(\operatorname{cl}\left(V_{0}\right)\right)$ is compact we can find an open neighbourhood $V_{0}^{\prime}$ of 0 in $V$ satisfying $V_{0}^{\prime} \subset V_{0}$ such that $d h(x)^{*_{r}}$ is injectiv for every $x \in \operatorname{cl}\left(V_{0}^{\prime}\right)$ and every $r \in[0,1]$. Set

$$
\epsilon^{\prime}:=\min _{v \in V \backslash V_{0}^{\prime}}\|v\|>0
$$

Since the family of injective maps $d h(x)^{*_{r}}$ depends smoothly on the compact parameter $(x, r) \in h^{-1}\left(\operatorname{cl}\left(V_{0}^{\prime}\right)\right) \times[0,1]$ there exists a compact subset $W \in V^{*}$ such that

$$
\left\|\nabla_{g_{M, r}} f_{r}(x)+d h(x)^{*_{r}} v^{*}\right\|_{g_{M, r}} \geq \epsilon^{\prime}, \quad v^{*} \in V^{*} \backslash W, x \in h^{-1}\left(\operatorname{cl}\left(V_{0}^{\prime}\right)\right), r \in[0,1] .
$$

We set

$$
K_{0}=h^{-1}\left(\operatorname{cl}\left(V_{0}^{\prime}\right)\right) \times W .
$$

Then $K_{0}$ is compact and we claim that

$$
\left\|\nabla_{g_{M, r}} f_{r}(x)+d h(x)^{*_{r}} v^{*}\right\|_{g_{M, r}}+\|h(x)\| \geq \epsilon^{\prime}, \quad y=\left(x, v^{*}\right) \in\left(M \times V^{*}\right) \backslash K_{0} .
$$

To prove the claim we first assume that $x \notin h^{-1}\left(\operatorname{cl}\left(V_{0}^{\prime}\right)\right)$. We then estimate

$$
\left\|\nabla_{g_{M, r}} f_{r}(x)+d h(x)^{*_{r}} v^{*}\right\|_{g_{M, r}}+\|h(x)\| \geq\|h(x)\| \geq \epsilon^{\prime}
$$


by the definition of $\epsilon^{\prime}$. Now assume that $x \in h^{-1}\left(\operatorname{cl}\left(V_{0}^{\prime}\right)\right)$ but $v^{*} \notin W$. We estimate in this case using (32)

$$
\left\|\nabla_{g_{M, r}} f_{r}(x)+d h(x)^{*_{r}} v^{*}\right\|_{g_{M, r}}+\|h(x)\| \geq\left\|\nabla_{g_{r}} f_{r}(x)+d h(x)^{*_{r}} v^{*}\right\|_{g_{M, r}} \geq \epsilon^{\prime}
$$

This proves (33).

Using (31) and (33) we estimate for $y=\left(x, v^{*}\right) \in\left(M \times V^{*}\right) \backslash K_{0}$

$$
\left\|\nabla_{g_{r}} F_{r}(y)\right\|_{g_{r}} \geq \frac{1}{\sqrt{2}}\left(\left\|\nabla_{g_{M, r}} f_{r}(x)+d h(x)^{*_{r}} v^{*}\right\|_{g_{M, r}}+\|h(x)\|\right) \geq \frac{\epsilon^{\prime}}{\sqrt{2}}>0
$$

which implies the first inequality in (30). To prove the second one we observe that since the metrics $g_{M, r}$ differ from $g_{M}$ only on a compact subset of $M$ the metrics $g_{M, r}$ are equivalent for every $r \in[0,1]$, i.e. there exists a constant $c \geq 1$ such that

$$
\frac{1}{c^{2}} g_{M} \leq g_{M, r} \leq c^{2} g_{M}, \quad r \in[0,1]
$$

Using (31), (33), and (34) we estimate for $y=\left(x, v^{*}\right) \in\left(M \times V^{*}\right) \backslash K_{0}$

$$
\begin{aligned}
\left\|\nabla_{g_{r}} F_{r}(y)\right\|_{g_{r}}^{2} & =\left\|\nabla_{g_{M, r}} f_{r}(x)+d h^{*_{r}}(x) v^{*}\right\|_{g_{M, r}}^{2}+\|h(x)\|^{2} \\
& \geq \frac{1}{2}\left(\left\|\nabla_{g_{M, r}} f_{r}(x)+d h^{* r}(x) v^{*}\right\|_{g_{M, r}}+\|h(x)\|\right)^{2} \\
& \geq \frac{\epsilon^{\prime}}{2}\left(\frac{1}{c}\left\|\nabla_{g_{M, r}} f_{r}(x)+d h^{* r}(x) v^{*}\right\|_{g_{M}}+\|h(x)\|\right) \\
& \geq \frac{\epsilon^{\prime}}{2^{3 / 2} c}\left\|\nabla_{g_{r}} F_{r}(y)\right\|_{g_{P S} .}
\end{aligned}
$$

Hence the second inequality in (30) follows with $\epsilon=\epsilon^{\prime} / 2^{3 / 2} c$. This proves Step 5 .

Step 6: We prove (iii).

Let $y \in \bigcup_{r \in[0,1]} \mathcal{M}\left(F_{r}, g_{r}\right)$. Let $K_{0} \subset M \times V^{*}$ be the compact set found in Step 5. We estimate for each $\sigma \in \mathbb{R}$ the distance $\operatorname{dist}_{P S}\left(y(\sigma), K_{0}\right)$ between $y(\sigma)$ and $K_{0}$ with respect to the Palais-Smale metric $g_{P S}$ found in Step 5 . We abbreviate

$$
m:=\max _{\substack{x \in K_{0}, r \in[0,1]}} F_{r}(x)-\min _{\substack{x \in K_{0}, r \in[0,1]}} F_{r}(x) .
$$

Since the Morse flow line $y$ has finite energy it follows from (30) that for each $\sigma \in \mathbb{R}$ the set $\left\{s \geq \sigma: y(s) \in K_{0}\right\}$ is nonempty. We set

$$
\tau(\sigma)=\inf \left\{s \geq \sigma: y(s) \in K_{0}\right\}
$$


Using (30) and the gradient equation we estimate

$$
\begin{aligned}
\operatorname{dist}_{P S}\left(y(\sigma), K_{0}\right) & \leq \int_{\sigma}^{\tau(\sigma)}\left\|\partial_{s} y(s)\right\|_{g_{P S}} d s \\
& =\int_{\sigma}^{\tau(\sigma)}\left\|\nabla_{g_{r}} F_{r}(y(s))\right\|_{g_{P S}} d s \\
& \leq \frac{1}{\epsilon} \int_{\sigma}^{\tau(\sigma)}\left\|\nabla_{g_{r}} F_{r}(y(s))\right\|_{g_{r}}^{2} d s \\
& \leq \frac{1}{\epsilon} \int_{-\infty}^{\infty}\left\|\nabla_{g_{r}} F_{r}(y(s))\right\|_{g_{r}}^{2} d s \\
& =-\frac{1}{\epsilon} \int_{-\infty}^{\infty} g_{r}(y(s))\left(\nabla_{g_{r}} F_{r}(y(s)), \partial_{s} y(s)\right) d s \\
& =-\frac{1}{\epsilon} \int_{-\infty}^{\infty} d F_{r}(y(s)) \partial_{s} y(s) d s \\
& =-\frac{1}{\epsilon} \int_{-\infty}^{\infty} \frac{d}{d s} F_{r}(y(s)) d s \\
& \leq \frac{1}{\epsilon}\left(\limsup _{s \rightarrow-\infty} F_{r}(y(s))-\liminf _{s \rightarrow \infty} F_{r}(y(s))\right) \\
& \leq \frac{m}{\epsilon} .
\end{aligned}
$$

We now set

$$
K:=\left\{y \in M \times V^{*}: \operatorname{dist}_{P S}\left(y, K_{0}\right) \leq \frac{m}{\epsilon}\right\} .
$$

Since $g_{P S}$ is geodesically complete, the set $K$ is compact. Moreover, the estimate above shows that

$$
\left\{y(\sigma): y \in \bigcup_{r \in[0,1]} \mathcal{M}\left(F_{r}, g_{r}\right), \sigma \in \mathbb{R}\right\} \subset K
$$

holds. This proves Step 6 .

Step 7: We prove the theorem

It remains to show that the functions $F_{r}$ are Morse-Bott. We prove that in Proposition A.2 below. This finishes the proof of the theorem.

If $x$ is a critical point of a Morse-Bott function, then we define the index $\operatorname{ind}_{F}(x)$ of $F$ at $x$ as the number of negative eigenvalues of the Hessian of $F$ at $x$. Note that the Morse-Bott condition implies that the index is constant on each connected component of $\operatorname{crit}(F)$. The following proposition shows that if $\left.f\right|_{h^{-1}(0)}$ is Morse-Bott, then the Lagrange multiplier functional is also MorseBott and its index is independent of the behaviour of $f$ outside of $h^{-1}(0)$. 
However note, that the Hessian itself depends also on the derivatives of $f$ in the normal directions of $h^{-1}(0)$.

Proposition A.2 Let $M$ be a finite dimensional manifold and let $(V,\langle\rangle$, be a $k$-dimensional Euclidean vector space. Assume that $f \in C^{\infty}(M), h \in$ $C^{\infty}(M, V)$ such that 0 is a regular value of $h$ and $\left.f\right|_{h^{-1}(0)}$ is Morse-Bott. Then $F \in C^{\infty}\left(M \times V^{*}\right)$ defined by $F\left(x, v^{*}\right)=f(x)+v^{*}(h(x))$ for $\left(x, v^{*}\right) \in M \times V^{*}$ is also Morse-Bott. Moreover, if $\lambda: \operatorname{crit}(F) \rightarrow \operatorname{crit}\left(\left.f\right|_{h^{-1}(0)}\right)$ is the natural bijection given by $\left(x, v^{*}\right) \mapsto x$ for $\left(x, v^{*}\right) \in \operatorname{crit}(F)$, then for the indices the following relation holds

$$
\operatorname{ind}_{F}\left(\lambda^{-1}(x)\right)=\operatorname{ind}_{\left.f\right|_{h^{-1}(0)}}(x)+k, \quad x \in \operatorname{crit}\left(\left.f\right|_{h^{-1}(0)}\right) .
$$

Proof: Let $x \in \operatorname{crit}\left(\left.f\right|_{h^{-1}(0)}\right)$. We first choose convenient coordinates around $x$ in $M$. Set $n=\operatorname{dim}(M)$ and chose $\delta_{1}, \delta_{2}>0$ so small such that there exists a diffeomorphism $\phi$ from $B_{\delta_{1}}^{n-k} \times B_{\delta_{2}}^{k}=\left\{v \in \mathbb{R}^{n-k}:\|v\|<\delta_{1}\right\} \times\left\{v \in \mathbb{R}^{k}:\|v\|<\right.$ $\left.\delta_{2}\right\}$ to an open neighbourhood $U$ of $x$ in $M$ such that

$$
\phi(0,0)=x, \quad h(\phi(q, w))=w, \quad q \in B_{\delta_{1}}^{n-k}, w \in B_{\delta_{2}}^{k} .
$$

Choose furthermore an orthonormal basis in $V^{*}$ to define an isomorphism $\Phi: V^{*} \rightarrow$ $\mathbb{R}^{k}$. Let $\hat{f} \in C^{\infty}\left(B_{\delta_{1}}^{n-k} \times B_{\delta_{2}}^{k}\right)$, be given by the pullback of $f$, i.e.

$$
\hat{f}=\left.\phi^{*} f\right|_{U},
$$

and $\hat{F} \in C^{\infty}\left(B_{\delta_{1}}^{n-k} \times B_{\delta_{2}}^{k} \times \mathbb{R}^{k}\right)$ be given by the pullback of $F$, i.e.

$$
\hat{F}=\left.(\phi \times \Phi)^{*} F\right|_{U \times V^{*}}
$$

Then $\hat{F}$ reads

$$
\hat{F}(q, w, v)=\hat{f}(q, v)+\langle v, w\rangle, \quad q \in B_{\delta_{1}}^{n-k}, v \in B_{\delta_{2}}^{k}, w \in \mathbb{R}^{k} .
$$

We choose the standard flat metric on $B_{\delta_{1}}^{n-k} \times B_{\delta_{2}}^{k} \times \mathbb{R}^{k}$ and introduce the $k \times k$-matrix $A$, the $k \times(n-k)$-matrix $B$ and the $(n-k) \times(n-k)$-matrix $H$ by

$$
A_{i j}=\frac{\partial^{2} \hat{f}(0,0)}{\partial w_{i} \partial w_{j}}, \quad B_{i j}=\frac{\partial^{2} \hat{f}(0,0)}{\partial q_{i} \partial w_{j}}, \quad H_{i j}=\frac{\partial^{2} \hat{f}(0,0)}{\partial q_{i} \partial q_{j}} .
$$

Denote by $\pi_{2}: M \times V^{*} \rightarrow V^{*}$ the projection to the second factor. The Hessian of $\hat{F}$ at $\left(0,0, \Phi \circ \pi_{2} \circ \lambda^{-1}(x)\right)$ with respect to the standard flat metric is given by

$$
H_{\hat{F}}\left(0,0, \Phi \circ \pi_{2} \circ \lambda^{-1}(x)\right)=\left(\begin{array}{ccc}
H & B & 0 \\
B^{T} & A & \text { id } \\
0 & \text { id } & 0
\end{array}\right)
$$

We claim that

$$
\operatorname{dim}\left(\operatorname{ker} H_{\hat{F}}\left(0,0, \Phi \circ \pi_{2} \circ \lambda^{-1}(x)\right)\right)=\operatorname{dim}(\operatorname{ker} H) .
$$


To see that assume that the vector $(\hat{q}, \hat{w}, \hat{v}) \in \mathbb{R}^{n-k} \times \mathbb{R}^{k} \times \mathbb{R}^{k}$ lies in the kernel of $H_{\hat{F}}\left(0,0, \Phi \circ \pi_{2} \circ \lambda^{-1}(x)\right.$. It follows that

$$
\left\{\begin{array}{c}
H \hat{q}+B \hat{w}=0 \\
B^{T} \hat{q}+A \hat{w}+\hat{v}=0 \\
\hat{w}=0
\end{array}\right.
$$

which implies that

$$
(\hat{q}, \hat{w}, \hat{v})=\left(\hat{q}, 0,-B^{T} \hat{q}\right), \quad \hat{q} \in \operatorname{ker}(H) .
$$

Hence (35) follows.

To prove that $F$ is Morse-Bott we denote for $y \in \operatorname{crit}(F)$ by $\operatorname{dim}_{y}(\operatorname{crit}(F))$ the local dimension at $y$ of the (unconnected) manifold $\operatorname{crit}(F)$ and compute using (35) and the Morse-Bott assumption on $\left.f\right|_{h^{-1}(0)}$

$$
\begin{aligned}
\operatorname{dim}\left(\operatorname{ker} H_{\hat{F}}\left(\lambda^{-1}(0)\right)\right. & =\operatorname{dim}\left(\operatorname{ker} H_{\left.f\right|_{h^{-1}(0)}}(x)\right) \\
& =\operatorname{dim}_{x}\left(\operatorname{crit}\left(\left.f\right|_{h^{-1}(0)}\right)\right) \\
& =\operatorname{dim}_{\lambda^{-1}(x)}(\operatorname{crit}(F)) .
\end{aligned}
$$

This proves that $F$ is Morse-Bott.

It remains to compute the index of the Hessian of $F$. To do that we consider the smooth family of functions $\hat{f}_{r} \in C^{\infty}\left(B_{\delta_{1}}^{n-k} \times B_{\delta_{2}}^{k}\right)$ for $r \in[0,1]$ defined by

$$
\hat{f}_{r}(q, w)=(1-r) \hat{f}(q, w)+r \hat{f}(q, 0), \quad q \in B_{\delta_{1}}^{n-k}, w \in B_{\delta_{2}}^{k} .
$$

Then

$$
\hat{f}_{0}=\hat{f},\left.\quad \hat{f}_{r}\right|_{B_{\delta_{1}}^{n-k} \times\{0\}}=\left.\hat{f}\right|_{B_{\delta_{1}}^{n-k} \times\{0\}}, r \in[0,1] .
$$

We define the smooth family of functions $\hat{F}_{r} \in C^{\infty}\left(B_{\delta_{1}}^{n-k} \times B_{\delta_{2}}^{k} \times \mathbb{R}^{k}\right)$ for $r \in[0,1]$ by

$$
\hat{F}_{r}(q, w, v)=\hat{f}_{r}(q, w)+\langle v, w\rangle .
$$

Define further the smooth family of vectors $v_{r} \in \mathbb{R}^{k}$ for $r \in[0,1]$ by

$$
\left(v_{r}\right)_{i}=-\frac{\partial \hat{f}_{r}(0,0)}{\partial w_{i}}, \quad i \in\{1, \ldots, k\}
$$

The functions $\hat{F}_{r}$ have critical points at $\left(0,0, v_{r}\right)$ and it follows from (35) that $\operatorname{dim}\left(\operatorname{ker} H_{\hat{F}_{r}}\left(0,0, v_{r}\right)\right)=\operatorname{dim}(\operatorname{ker}(H))$ does not depend on $r \in[0,1]$. Since the eigenvalues of a continuous family of matrices are continuous, see [18, Theorem II.5.1] we conclude

$$
\operatorname{ind}_{\hat{F}_{0}}\left(0,0, v_{0}\right)=\operatorname{ind}_{\hat{F}_{1}}\left(0,0, v_{1}\right) .
$$

The Hessian of $\hat{F}_{1}$ at $\left(0,0, v_{1}\right)=(0,0,0)$ is given by

$$
H_{\hat{F}_{1}}(0,0,0)=\left(\begin{array}{ccc}
H & 0 & 0 \\
0 & 0 & \text { id } \\
0 & \text { id } & 0
\end{array}\right)
$$


from which we deduce

$$
\operatorname{ind}_{\hat{F}_{1}}\left(0,0, v_{1}\right)=\operatorname{ind}_{\hat{f}_{B_{\delta_{1}}^{n-k} \times\{0\}}}(0,0)+k .
$$

Combining (36) and (37) we compute

$$
\begin{aligned}
\operatorname{ind}_{F}\left(\lambda^{-1}(x)\right) & =\operatorname{ind}_{\hat{F}_{0}}\left(0,0, v_{0}\right) \\
& =\operatorname{ind}_{\hat{f}_{B_{\delta_{1}}^{n-k} \times\{0\}}}(0,0)+k \\
& =\operatorname{ind}_{\left.f\right|_{h^{-1}(0)}}(x)+k .
\end{aligned}
$$

This completes the proof of the proposition.

\section{References}

[1] S. Bradlow, Vortices in holomorphic line bundles over closed Kähler manifolds, Comm. Math. Phys. 135 (1990), no 1, 1-17.

[2] K. Cieliebak, R. Gaio, I. Mundet, D. Salamon, The symplectic vortex equations and invariants of Hamiltonian group actions, J. Symplectic Geom. 1 (2002), no. 3, 543-645.

[3] K. Cieliebak, R. Gaio, D. Salamon, J-holomorphic curves, moment maps, and invariants of Hamiltonian group actions, Int. Math. Res. Not. (2000), no. $16,831-882$.

[4] K. Cieliebak, I.Mundet, D. Salamon, Equivariant moduli problems, branched manifolds, and the Euler class, Topology 42(2003), no. 3, 641700 .

[5] W. Ding, J. Jost, J. Li, X. Peng, G. Wang, Self Duality Equations for Ginzburg-Landau and Seiberg-Witten Type Functionals with $6^{\text {th }}$ Order Potential, Commun. Math. Phys. 217, 383 -407 (2001).

[6] S. Dostoglou, D. Salamon, Self-dual instantons and holomorphic curves, Annals of Math. 139 (1994), 581-640.

[7] U. Frauenfelder, The Arnold-Givental conjecture and moment Floer homology, Int. Math. Res. Not. (2004), no 42, 2179-2269.

[8] K. Fukaya, K. Ono, Arnold conjecture and Gromov-Witten invariants for general symplectic manifolds, Topology 38 (1999), 933-1048.

[9] M. Furuta, Monopole equation and the 11/8-conjecture, Math. Res. Lett, 8 (2001), 279-291.

[10] O. Garcia-Prada, A direct existence proof for the vortex equations over a compact Riemann surface, Bull. London Math. Soc. 26 (1994), no 1, 88-96. 
[11] A. Givental, Homological Geometry. I. Projective Hypersurfaces, Selecta Math. (N.S.) 1 (1995), no.2, 325-345.

[12] A. Givental, A Mirror Theorem for Toric Complete Intersections. Topological field theory, primitive forms and related topics (Kyoto, 1996), pp.141175, Progr. Math., 160, Birkhäuser Boston, Boston, MA, 1998.

[13] M. Greenberg, J. Harper, Algebraic topology, Mathematics Lecture Note Series 58 (1981).

[14] J. Hong, Y. Kim, P. Pac, Multivortex solutions of the Abelian Chern-Simons theory, Phys. Rev. Lett. 64, 2230-2233 (1990).

[15] H. Iritani, Quantum D-module and equivariant Floer theory for free loop spaces, preprint, math.DG/0410487.

[16] R. Jackiw, E. Weinberg, Self-dual Chern-Simons vortices, Phys. Rev. Lett. 64, 2234-2237 (1990).

[17] A. Jaffe, C. Taubes, Vortices and monopoles, Progress in Physics 2 (1980).

[18] T. Kato, Perturbation theory for linear operators, Springer, Berlin (1976).

[19] J.Kazdan, F. Warner, Curvature functions for compact 2-manifolds, Ann. Math. 99 (1978), no 2, 14-47.

[20] P. Kronheimer, C. Manolescu, Periodic Floer Pro-Spectra from the SeibergWitten equations, preprint, math.GT/0203243

[21] J.Li, G. Tian, Virtual moduli cycles and $G W$ invariants of general symplectic manifolds, in [26], 47-83.

[22] G. Liu, G. Tian, Floer homology and Arnold conjecture, J. Diff. Geom. 49 (1998), 1-74.

[23] C. Manolescu, Seiberg-Witten-Floer stable homotopy type of three-manifolds with $b_{1}=0$, preprint, math.DG/0104024

[24] Y.Ruan, Virtual neighbourhoods and pseudoholomorphic curves, in [26], $101-116$.

[25] B. Siebert, Symplectic Gromov-Witten invariants, in: New Trends in Algebraic Geometry, ed. Catanese, Peters, Reid, L.M.S. Lect. Notes 264, Camb. Univ. Press (1999), 375-424.

[26] R.Stern, Topics in Symplectic 4-manifolds (Irvine CA 1996), Internat. Press Lecture Series, Vol 1, Internat. Press, Cambridge, MA (1998).

[27] G. Tarantello, Multiple condensate solutions for the Chern-Simon-Higgs theory, J. Math. Phys. 37, 3769-3796 (1996). 
[28] Y. Vlassopoulos, Quantum Cohomology and Morse Theory on the Loop Space of Toric Varieties, preprint, math.AG/0203083.

[29] Y. Yang, Solitons in field theory and nonlinear analysis, Springer, New York (2001). 